\title{
Self-Excited and Hidden Chaotic Attractors in Matouk's Hyperchaotic Systems
}

\author{
A. Othman Almatroud $\mathbb{D},{ }^{1}$ A. E. Matouk $\left(\mathbb{D},{ }^{2,3}\right.$ Wael W. Mohammed ${ }^{D},{ }^{1,4}$ Naveed Iqbal $\left(\mathbb{D},{ }^{1}\right.$ \\ and Saleh Alshammari ${ }^{1}$ \\ ${ }^{1}$ Department of Mathematics, Faculty of Science, University of Ha'il, Ha'il 2440, Saudi Arabia \\ ${ }^{2}$ Department of Mathematics, College of Science Al-Zulfi, Majmaah University, Al-Majmaah 11952, Saudi Arabia \\ ${ }^{3}$ College of Engineering, Majmaah University, Al-Majmaah 11952, Saudi Arabia \\ ${ }^{4}$ Department of Mathematics, Faculty of Science, Mansoura University, Mansoura 35516, Egypt
}

Correspondence should be addressed to A. E. Matouk; aematouk@hotmail.com

Received 6 August 2021; Revised 30 November 2021; Accepted 21 January 2022; Published 8 March 2022

Academic Editor: Rigoberto Medina

Copyright (c) $2022 \mathrm{~A}$. Othman Almatroud et al. This is an open access article distributed under the Creative Commons Attribution License, which permits unrestricted use, distribution, and reproduction in any medium, provided the original work is properly cited.

\begin{abstract}
Self-excited and hidden chaotic attractors are interesting complex dynamical phenomena. Here, Matouk's hyperchaotic systems are shown to have self-excited and hidden chaotic attractors, respectively. Two case studies of hidden chaotic attractors are provided which are examined with orders 3.08 and 3.992, respectively. Moreover, self-excited chaotic attractors are found in the fractional-order system and its integer-order counterpart. The existence of one-eyed face self-excited chaotic attractors is also reported in this work. Our results show that the fractional derivative affects the appearances of hidden chaotic attractors in this system.
\end{abstract}

\section{Introduction}

Recently, fractional-order calculus (FC) has been utilized to describe complex dynamics arising from nonlinear dynamical systems (NDS) [1-8]. FC also has some interdisciplinary applications in several scientific fields. For example, it is widely used in engineering [9], heat conduction, and thermoelectricity models $[10,11]$.

In fact, self-excited attractors and hidden attractors are very interesting complex dynamical phenomena that can be found in some NDS involving fractional- or integer-order derivatives [12, 13]. The basin of attraction of a hidden attractor is not connected with small neighborhoods of any stationary state (equilibrium point) unlike the case of selfexcited attractors. Therefore, hidden attractors occur in systems with unique stable stationary (equilibrium) states or with no equilibria. One of the famous problems dealing with the hidden attractors is studying mutual disposition and the number of limit cycles in 2D polynomial differential systems $[14,15]$. The former problem had firstly been arisen by
Hilbert in 1900, in association with Hilbert's sixteenth problem $\left(2^{\text {nd }}\right.$ part), to investigate hidden periodic oscillations [16]. Hidden attractors had also been arisen in the study of Aizerman and Kalman conjectures. It is found that hidden attractors have potential applications in many fields like ecosystems, climate science, physics, engineering, financial market, aircraft control systems, and phase-locked loop-based circuits. Recently, some NDS involving hidden attractors with stable equilibria and without equilibrium points have appeared [17-22]. For example, Yang et al. observed hidden chaotic attractors in a system with two stable node foci [17]; Dudkowski et al. found hidden chaotic attractors in Chua's circuit [18]; Munmuangsaen and Srisuchinwong reported hidden chaotic attractors in the wellknown Lorenz model for atmospheric convection [19]; and Kuznetsov and Leonov studied hidden attractors in some engineering and fundamental problems [20,21]. Singh and Roy obtained hidden attractors in a new $4 \mathrm{D}$ chaotic system involving a line of equilibria [22]. Furthermore, the interesting phenomena of coexisting self-excited attractors (CS- 
EAs) occur in a system when several attractors with independent basin sets of attraction take place with different initial conditions for a given parameter set. The CS-EA is usually determined via the standard computational procedures. In [23], Singh and Roy showed the coexistence of selfexcited chaotic attractors in a new $3 \mathrm{D}$ chaotic system.

In this work, we mainly focus on exploring hidden chaotic attractors and self-excited coexisting attractors in a novel hyperchaotic system given by Matouk [24]. The proposed system is described by a set of four coupled nonlinear integer or fractional-order differential equations [25] and is known here as Matouk's hyperchaotic system. Matouk's system was applied to solve some potential engineering problems $[3,24]$. Here, the novel dynamical behaviors of Matouk's hyperchaotic systems have the following different types: (i) hidden chaotic attractors exist in the integer-order and the fractional-order Matouk's hyperchaotic systems with one saddle equilibrium point and two locally asymptotically stable equilibria; (ii) variety of selfexcited chaotic attractors exist in this system including the newly found one-eyed face attractors; (iii) coexistence of multihidden attractors, with different topological shapes, are also found in this system.

The organization of this paper is described as follows: Section 2 is devoted to represent the basic notations related to the fractional analysis used in this work. In Section 3, we present some dynamical behaviors of Matouk's hyperchaotic system including some stability conditions of its equilibrium solutions and some conditions for its chaoticity based on Shil'nikov's Theorem. In Section 4, we investigate the hidden chaotic attractors and self-excited chaotic attractors that existed in Matouk's systems. Finally, this work is ended by a conclusion in Section 5.

\section{Preliminaries}

The Caputo fractional differential operator [26] can be expressed by

$$
\mathrm{Cap} D_{0}^{\eta} \omega(t)=\frac{\left[\int_{0}^{t}(t-\gamma)^{p-\eta-1} \omega^{(p)}(\gamma) \mathrm{d} \gamma\right]}{\Gamma(p-\eta)},
$$

where $\eta \in R^{+}$and $\omega^{(p)}$ represents the $p$ th-order derivative of $\omega(\gamma)$ such that $p-1<\eta<p \in Z^{+}$. Obviously, this operator is nonlocal with a singular kernel. Therefore, it is convenient to describe the complex dynamics arising from the NDS.

Consider the autonomous system

$$
\frac{\mathrm{d}^{\eta} \mathrm{Y}}{\mathrm{d} t^{\eta}}=f(\mathrm{Y})
$$

where $0<\eta \leq 1$ and $f$ is a nonlinear vector function. If $J$ represents the Jacobian of the linearized counterpart of autonomous system (2), then the local stability of an equilibrium point $\bar{\Upsilon}$ of system (2) is governed by the following Matignon's inequalities:

$$
\eta<\frac{2}{\pi}\left|\arg \left(\varsigma_{j}\right)\right|, ; j=1, \ldots, n,
$$

where $\varsigma_{j}$ is an arbitrary eigenvalue of $J(\overline{\mathrm{Y}})$.
Throughout this work, we will refer by LAS an equilibrium point $\overline{\mathrm{Y}}$ that is locally asymptotically stable; i.e., all the eigenvalues of $\bar{Y}$ satisfies conditions (3).

\section{The Systems' Description}

Matouk's system with caputo fractional operators [25] is modeled by the following set of equations:

$$
\begin{aligned}
& \mathrm{Cap}_{0}^{\alpha} y_{1}=a\left(y_{4}-y_{2}\right)+h y_{1}-y_{1} y_{4}, \\
& \mathrm{Cap}_{0}^{\alpha} y_{2}=b y_{1}+y_{4}-y_{1} y_{3}, \\
& \mathrm{Cap}_{0}^{\alpha} y_{3}=y_{1}^{2}-c y_{3}, \\
& \mathrm{Cap}_{0}^{\alpha} y_{4}=\mathrm{d} y_{4}
\end{aligned}
$$

where the parameter set $A=\{a, b, c, d, h\} \subset R$ and $\alpha \in(0,1]$. System (4) is simple in comparison with similar types of $4 \mathrm{D}$ systems and reports studies of important problems $[3,24,25]$. It has the equilibria

$$
\begin{aligned}
& E q^{(1)}=(0,0,0,0), \\
& E q^{(2)}=\left(\sigma, \frac{\sigma h}{a}, b, 0\right), \\
& E q^{(3)}=\left(-\sigma,-\frac{\sigma h}{a}, b, 0\right), \quad \sigma=\sqrt{b c} .
\end{aligned}
$$

Assume that $\varsigma_{i}$ is an eigenvalue of the Jacobian matrix $J$ computed after the linearization of Matouk's system (4) and $J$ is evaluated at any of the equilibrium points described in equations (5). For $\alpha=1, E q^{(1)}$ has the following eigenvalues:

$$
\varsigma_{1}=-c, \varsigma_{2}=d, \varsigma_{3,4}=\frac{h \pm \sqrt{h^{2}-4 a b}}{2} .
$$

Thus, $E q^{(1)}$ is LAS if and only if

$$
a b \in R^{+}, c \in R^{+}, d \in R^{-}, h \in R^{-} \text {. }
$$

The other equilibrium points $E q^{(2)}$ and $E q^{(3)}$ have the same characteristic equation:

$$
(\varsigma-d)\left(\varsigma^{3}+\rho_{1} \varsigma^{2}+\rho_{2} \varsigma+\rho_{3}\right)=0
$$

where

$$
\rho_{1}=(c-h), \rho_{2}=-h c, \rho_{3}=-2 a b c .
$$

According to the classic Routh-Hurwitz criterion and the stability theory of integer-order differential systems, the equilibrium states $E q^{(2)}$ and $E q^{(3)}$ are LAS if and only if

$$
d<0, \rho_{1}>0, \rho_{3}>0, \rho_{1} \rho_{2}>\rho_{3} .
$$

For $\alpha \in(0,1]$ and according to Theorem 2 in Ref. [25], the original equilibrium point $E_{q}^{(1)}$ satisfies the following cases:

(1) $E_{q}^{(1)}$ is LAS inside the interval $\alpha \in(0,1]$ when $c \in R^{+}$and $d \in R^{-}$provided that

(i) $h \in R^{-}, a b \in R^{+}$ 
(ii) $a b>h^{2} / 4 ; a b \in R^{+} ; \alpha<2 / \pi \tan ^{-1}\left(\sqrt{4 a b-h^{2}} / h\right)$

(2) $E_{q}^{(1)}$ is saddle if $d c \in R^{+}$or $a b \in R^{-}$and the parameter $h$ has not vanished

Also, the local stability of the other nonorigin equilibrium points is determined by Matignon's conditions (3) or by testing the conditions of Theorem 3 in Ref. [25].

To discuss the chaoticity in system (4), we firstly assume that this system, with $\alpha=1$, has a saddle foci equilibria type in which the eigenvalues satisfy one of the following three cases:

(A) $\varsigma_{1,2}=-v \pm i \overline{\mathrm{\omega}}, \varsigma_{3} \in R^{+}$, and $\varsigma_{4} \leq 0$, where $\overline{\bar{\omega}}$ is not vanished and $v \in R^{+}$such that $\varsigma_{3}-v \in R^{+}$

((B) $\varsigma_{1,2}=v \pm i \bar{\Phi}, \varsigma_{3} \in R^{-}$, and $\varsigma_{4} \leq 0$, where $\bar{\omega}$ is not vanished and $v \in R^{+}$such that $\varsigma_{3}+v \in R^{-}$

(C) $\varsigma_{1,2}=v_{1} \pm i \bar{\omega}_{1}$ and $\varsigma_{3,4}=-v_{2} \pm i \bar{\Phi}_{2}$, where $\bar{\Phi}_{1}$ and $\bar{\Phi}_{2}$ are not vanished and $v_{1}, v_{2} \in R^{+}$such that $v_{1} \neq v_{2}$.

Then, we introduce the following theorems $[27,28]$.

Theorem 1. Assume that the original equilibrium state $E q^{(1)}$ satisfies one of conditions $(A-C)$, and assume that $E q^{(1)}$ is connected to itself by a homoclinic orbit. Then, the integerorder form of system (4) has Smale horseshoe chaos if eigenvalues (6) of $E q^{(1)}$ satisfy any of the following conditions:
(I) $\varsigma_{3}-2 v \in R^{-}$and conditions (A) hold
(II) $\varsigma_{3}+2 v \in R^{-}$and conditions (B) hold
(III) Conditions (C) hold

Theorem 2. Assume that the nonorigin equilibrium states $E q^{(2)}$ and $E q^{(3)}$ satisfy one of conditions $(A-C)$, and also assume that $E q^{(2)}$ and $E q^{(3)}$ are connected with heteroclinic orbits. Then, the integer-order form of system (4) has Smale horseshoe chaos if the eigenvalues of (8) satisfy any of the following conditions:
(I) $\varsigma_{3}-2 v \in R^{-}$and conditions (A) hold
(II) $\varsigma_{3}+2 v \in R^{-}$and conditions (B) hold
(III) Conditions (C) hold

For example, the parameter selection $a=12, b=15, c=7.35, d=-0.85, h=-0.95, \quad$ and $\quad \alpha=1$ fulfills condition (II) of Theorem 1 since the eigenvalues of the saddle-focus origin equilibrium state $E q^{(1)}$ satisfy this condition. Figure 1 shows the existence of homoclinic orbits shrinking to the origin state $E q^{(1)}$ for the aforementioned selection of the parameter values.

\section{Hidden and Self-Excited Chaotic Attractors in Matouk's System}

In this part, we will discuss the occurrence of hidden chaotic attractors and self-excited chaotic attractors in Matouk's systems (4). Indeed, the computational classification of an attractor is divided into two main types: hidden and self-

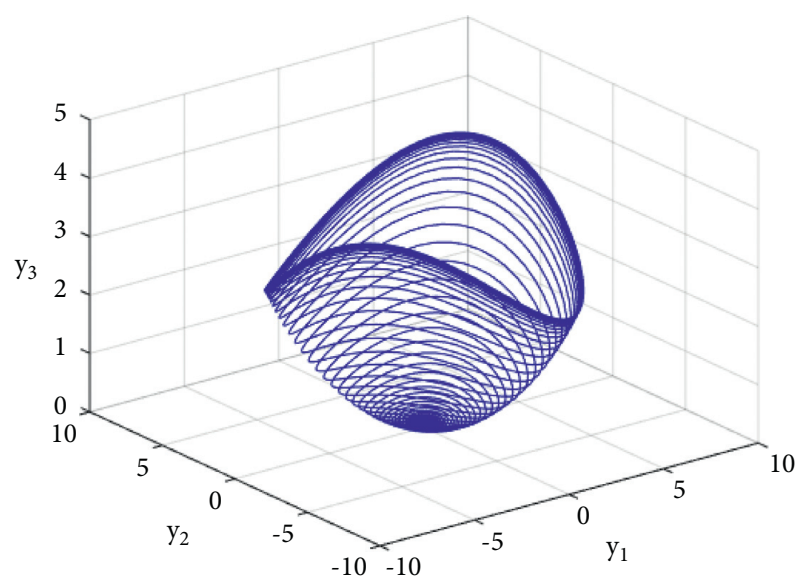

Figure 1: 3D view of the integer-order Matouk's system (4) with $a=12, b=15, c=7.35, d=-0.85, h=-0.95$.

excited attractors. These types can clearly be defined as follows.

Definition 1. (see [12]). A self-excited attractor is an attractor whose basin of attraction intersects with any open neighborhood of an equilibrium (stationary) state; otherwise, the attractor is said to be a hidden attractor.

Thus, the basin of attraction of a hidden attractor is not connected with stationary states (equilibria).

In [29], Prakash et al. pointed out that hyperchaotic systems can also be classified into two main types: (i) hidden attractors and (ii) self-excited attractors.

Matouk's equations (4) are coded based on the PECE (Predict, Evaluate, Correct, Evaluate) numerical scheme $[30,31]$. These codes are executed to obtain attractors with different topological shapes involving a variety of dynamical behaviors such as hidden and self-excited chaotic attractors and coexisting multihidden attractors.

Indeed, there exist different techniques to locate hidden attractors such as the method of perpetual points [32, 33]. To illustrate the hidden chaotic attractors, the PECE scheme is employed to obtain sets of 100 trajectories initiating from the unstable steady state. However, to visualize the hidden chaotic attractor, only representative trajectories have been depicted.

In the following, we will show the existence of different types of hidden and self-excited chaotic attractors in Matouk's equations (4) with fractional orders.

4.1. The First Case Study. In this section, the PECE method is used to obtain the numerical solution of the fractional Matouk's system with step size 0.005 . Using the parameter set $A_{1}=\{-3,15,0.6,-0.0001,-1.5\}$, the system's nonorigin equilibrium points are

$$
\begin{aligned}
& E q^{(2)}=(3,1.5,15,0), \\
& E q^{(3)}=(-3,-1.5,15,0),
\end{aligned}
$$


with eigen-spectrum $\Lambda=\{-0.0001, \quad-4.531316699$, $1.21565835 \pm 3.230981389 i\}, i=\sqrt{-1}$. So, they are LAS when $\alpha=0.77$ since they satisfy Matignon's stability conditions (3). Meanwhile, the original equilibrium point $E q^{(1)}$ is a saddle for any $\alpha \in(0,1]$, since it satisfies stability condition (2) of Section 3. Moreover, it has the eigenspectrum $\Lambda=\{6,-0.0001,-0.6,-7.5\}$ which means that it has an index one type of saddleness.

To explain the occurrence of the hidden chaotic attractors in the fractional case of system (4), we will show that trajectories originate from small neighborhoods of the unstable origin state $E q^{(1)}$ and are all attracted by the nonorigin LAS points $E q^{(2)}$ and $E q^{(3)}$. The results are depicted in Figure 2 in which the hidden chaotic attractor has the green color that surrounds the emanating trajectories from the close vicinity of the original state $E q^{(1)}$ which either approach the nonorigin state $E q^{(3)}=(-3,-1.5,15,0)$ (blue plot) or tend to the nonorigin state $E q^{(2)}=(3,1.5,15,0)$ (red plot). Thus, the selection of the initial conditions $\left(y_{10}, y_{20}, y_{30}, y_{40}\right)=(0.1,0,1,0.01)$ leads to a chaotic attractor whereas, the initial conditions $\left(y_{10}, y_{20}, y_{30}, y_{40}\right)=$ $(3,1,15,0.0199)$ and $\left(y_{10}, y_{20}, y_{30}, y_{40}\right)=(-3,-1,15,0.0199)$ lead to the red nonchaotic (asymptotic) attractor of $E q^{(2)}$ and the blue nonchaotic (asymptotic) attractor of $E q^{(3)}$, respectively. Thus, according to the abovementioned definition, a hidden chaotic attractor (the green domain in Figure 2) emerges if there exists another region from which this motion is chaotic. Obviously, the hidden chaotic attractor occurs when the order is $4 \times 0.77=3.08$.

Obviously, the fractional derivative affects the stability of the fractional form of system (4) according to Matignon's inequalities (3) and the abovementioned discussion. However, when $\alpha=1$, the nonorigin states $E q^{(2)}$ and $E q^{(3)}$ lose their stability and the corresponding two LAS attractors are replaced by two unstable focus-node points with an index 2 (saddle foci). However, $E q^{(1)}$ remains a saddle point of an index 1. Clearly, conditions (II) of Theorem 2 hold in this case. Then, if there exists a heteroclinic orbit that connects $E q^{(2)}$ and $E q^{(3)}$, system (4) exhibits a Smale horse-type chaos. Accordingly, the occurrence of chaotic attractors is expected. Figure 3 shows the existence of a self-excited chaotic attractor using the abovementioned selection of initial conditions along with the parameter set $A_{1}$ and $\alpha=1$. The computation of the corresponding basin set of attraction is illustrated in Figure 4.

The bifurcation diagrams corresponding to the abovementioned sets of initial conditions are illustrated in Figure 5. Moreover, Figure 5(c) shows that hidden chaotic attractors in system (4) exist for the selection of the parameter set $A_{1}$ where $c \in(0.36,0.65]$ and $\alpha=0.77$. Also, according to the selection of parameters in Figure 5(c), a new shape of self-excited chaotic attractors, one-eyed face, is found when $c=0.30,0.32,0.33,0.34,0.35$ and using different selections of initial conditions. The calculations of the corresponding spectrum of Lyapunov exponents $\lambda_{i, s}$ (or LEs) are depicted in Figure 6. Also, a variety of self-excited chaotic attractors in the fractional system are depicted in Figure 7 where trajectories with initial conditions in neighborhoods of the nonorigin points $E q^{(2)}$ and $E q^{(3)}$ (red domain) and (blue domain), respectively, tend to the chaotic attractor (green domain) surrounding the saddle origin point $E q^{(1)}$. Appearance of the newly found one-eyed face self-excited chaotic attractors is depicted in Figure 8. Hence, according to [34], system (4) fulfills the standard criteria for a new chaotic system. Furthermore, the coexistence of multihidden attractors is given in Figure 9 where their basins of attraction do not intersect with a certain neighborhood of the equilibrium points. Obviously, Figure 9 shows hidden attractors with different topological shapes such as coexistence of unstable saddle-focus attractors, coexistence of homoclinic orbits, coexistence of invariant closed curves, coexistence of higher periodic orbits, and existence of strange hidden chaotic attractors.

Obviously, Figure 5(e) shows that Matouk's system still exhibits chaotic attractors for a wide range as the derivative order is varied $(0.77 \leq \alpha \leq 1)$. This interesting result has also never been shown in an existing fractional system.

4.2. The Second Case Study. In this case, the PECE method is also used with step size 0.005 along with the set of parameters $A_{2}=\{-2,15,0.45,-0.15,-7.775\}$; the system's nonorigin equilibrium points are

$$
\begin{aligned}
& E q^{(2)}=(2.5981,10.1,15,0), \\
& E q^{(3)}=(-2.5981,-10.1,15,0) .
\end{aligned}
$$

For $\alpha \in(0,1]$, the nonorigin points are LAS since they satisfy stability conditions (3) and (9). However, the origin point $E q^{(1)}$ is the saddle of an index 1. This case also fulfills conditions (II) of Theorem 2. Hence, if there exists a heteroclinic orbit that connects $E q^{(2)}$ and $E q^{(3)}$, the integerorder case of system (4) exhibits a Smale horse-type chaos.

Also, in this case, a hidden chaotic attractor for system (4), $\alpha \in(0,1]$, is created by trajectories starting from the close vicinity of the origin point $E q^{(1)}$ that either approaches $E q^{(2)}=(2.5981,10.1,15,0)$ or $E q^{(3)}=(-2.5981,-10.1$, $15,0)$. Hence, the choice of initial data $\left(y_{10}, y_{20}, y_{30}, y_{40}\right)=$ $(0.01,0.01,0.01,0.01)$ leads to the chaotic attractor (green domain) whereas, the other selections $\left(y_{10}, y_{20}, y_{30}, y_{40}\right)=$ $(2.7,10.6,15,0.0001)$ and $\left(y_{10}, y_{20}, y_{30}, y_{40}\right)=(-2.7,-10.6$, $15,0.0001)$ lead to the asymptotic attractor of $E q^{(2)}$ (red domain) and the asymptotic attractor of $E q^{(3)}$ (blue domain), respectively. These hidden chaotic attractors are depicted in Figures 10 and 11, for the integer-order $(\alpha=1)$ and the fractional-order $(\alpha=0.998)$ cases, respectively. Consequently, in this case, the hidden chaotic attractor exists as the order is $4 \times 0.998=3.992$. The computation of a basin set of attraction corresponding to Figure 10 is illustrated in Figure 12. Moreover, computations of the corresponding bifurcation diagrams are illustrated in Figures 13 and 14 for $\alpha=1$ and $\alpha=0.998$, respectively. 


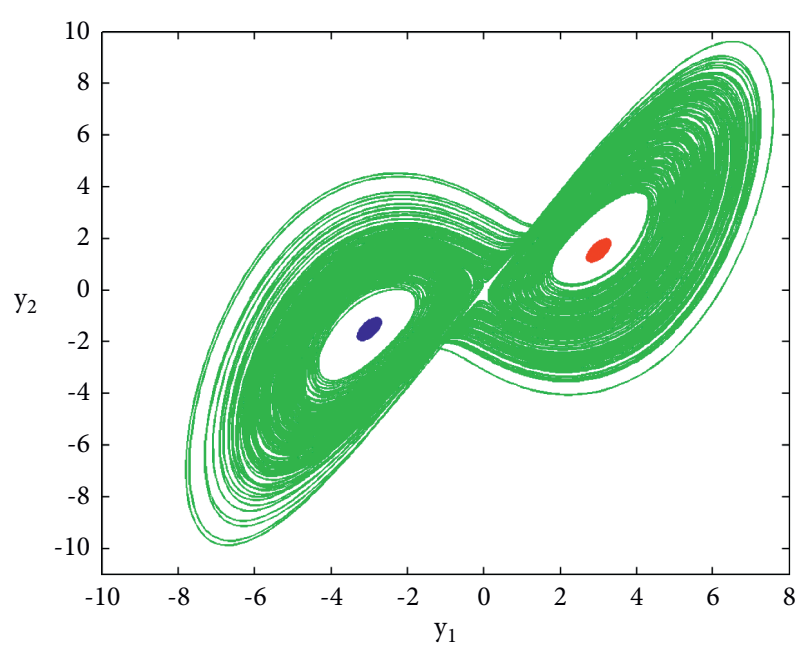

(a)

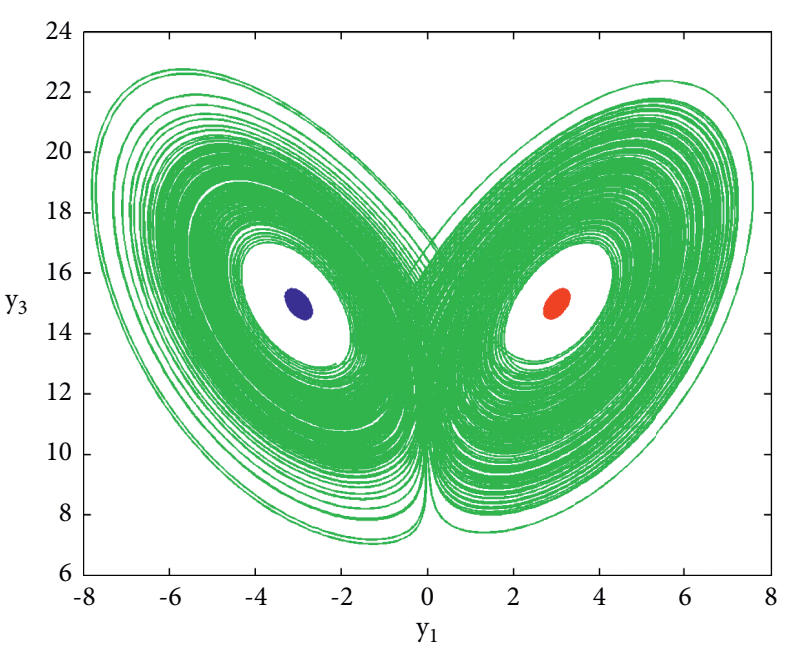

(b)

FiguRE 2: Hidden chaotic attractor (green plot) of system (4), with parameter selection $A_{1}$ and $\alpha=0.77$, showing the one-point attractor corresponding to $E q^{(2)}=(3,1.5,15,0)$ (red plot) and one-point attractor corresponding to $E q^{(3)}=(-3,-1.5,15,0)\left(\right.$ blue plot): $\left(\right.$ a) $y_{1} y_{2}$ view; (b) $y_{1} y_{3}$-view.

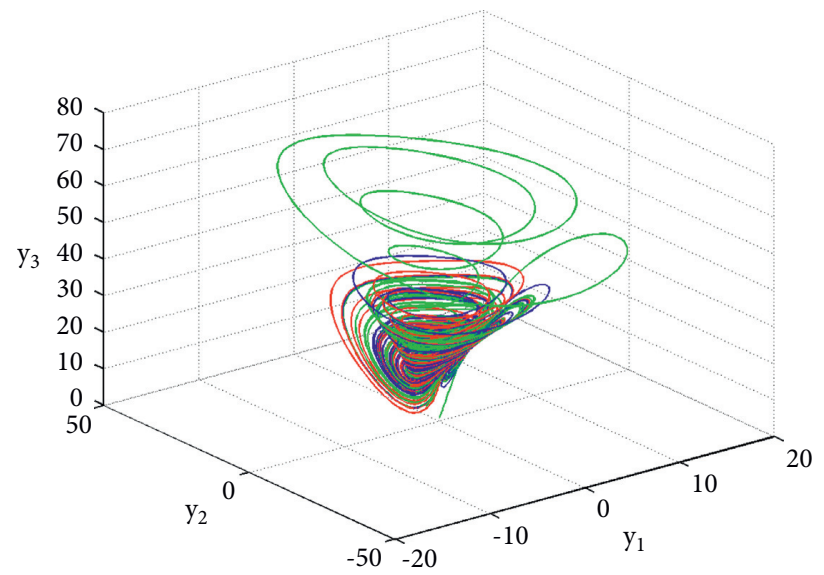

Figure 3: 3D plot of a self-excited chaotic attractor in the integer-order Matouk's system using the parameter set $A_{1}$ and initial conditions $(0.1,0,1,0.01)$ for green trajectory, $(3,1,15,0.0199)$ for red trajectory, and $(-3,-1,15,0.0199)$ for blue trajectory.

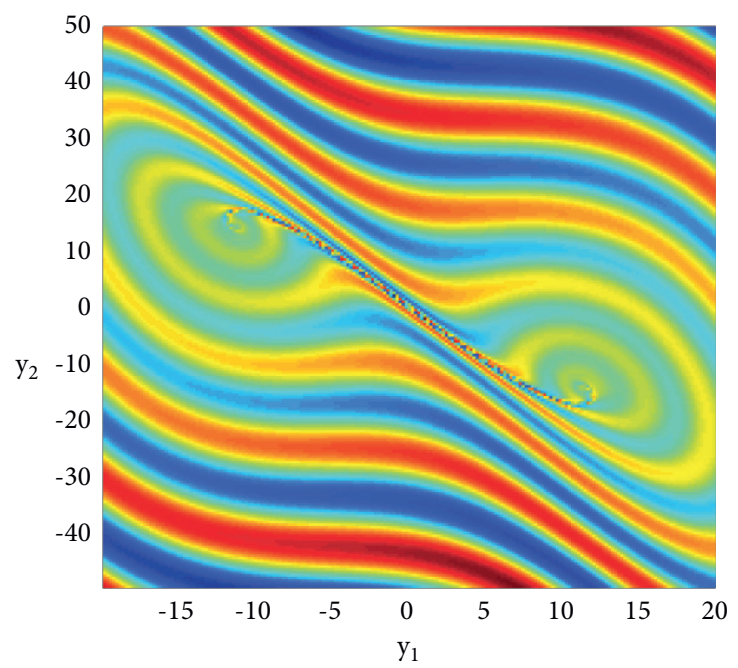

FIgURE 4: A cross section for $y_{3}=0$ and $y_{4}=0$ of basins of attraction for the self-excited attractor. 


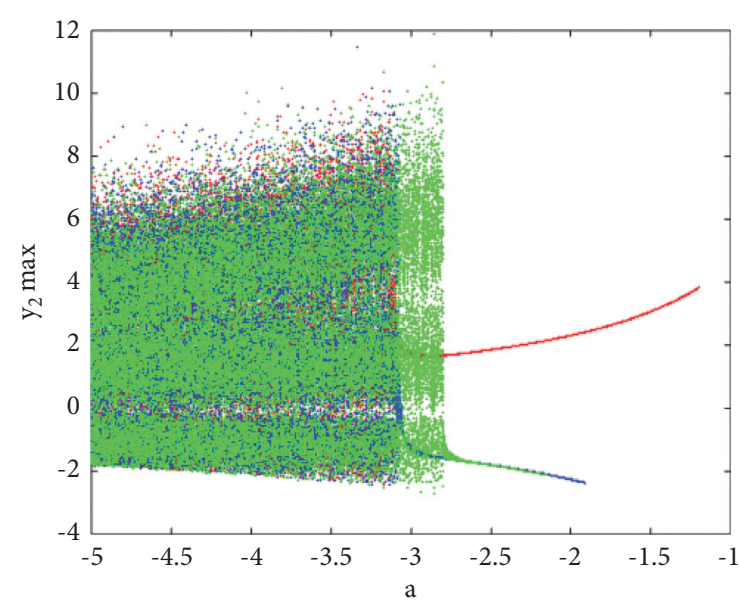

(a)

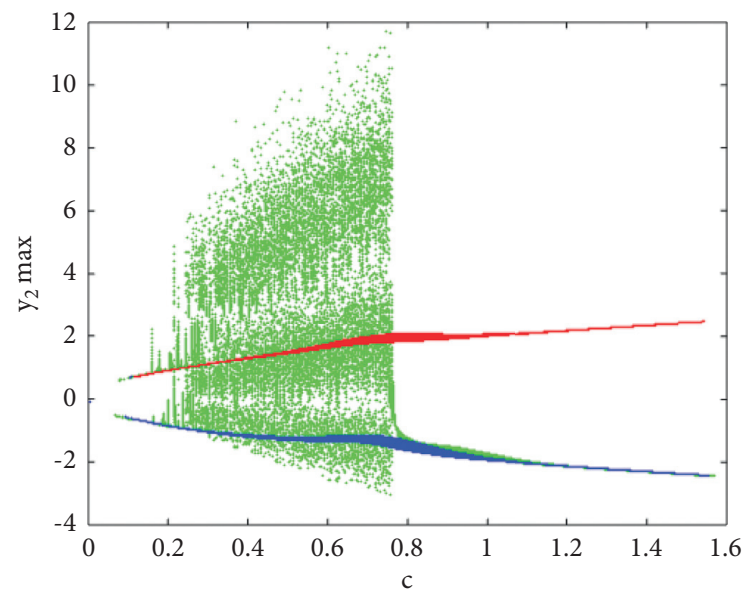

(c)

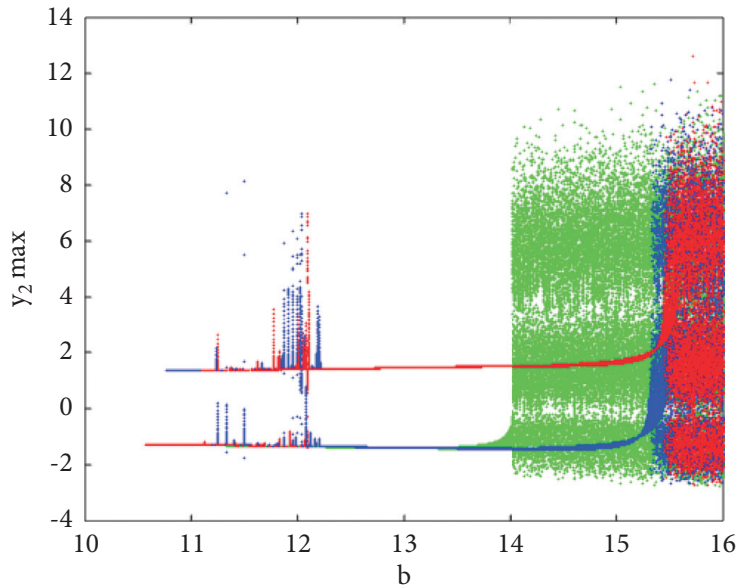

(b)

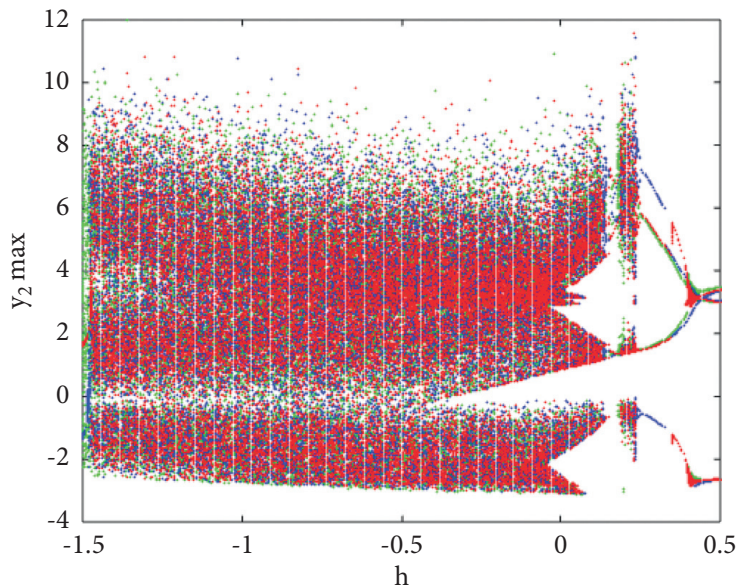

(d)

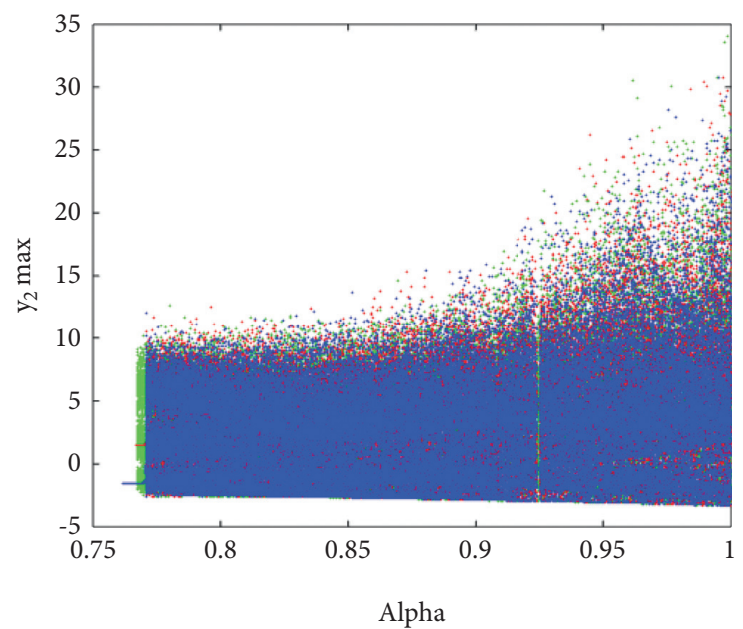

(e)

FIgURE 5: The bifurcation diagrams of the fractional-order Matouk's system with varying system's parameters, using the parameter set $A_{1}$ and $\alpha=0.77$ and initial conditions $(0.1,0,1,0.01)$ (green plot); $(3,1,15,0.0199)$ (red plot), and $(-3,-1,15,0.0199)($ blue plot). 


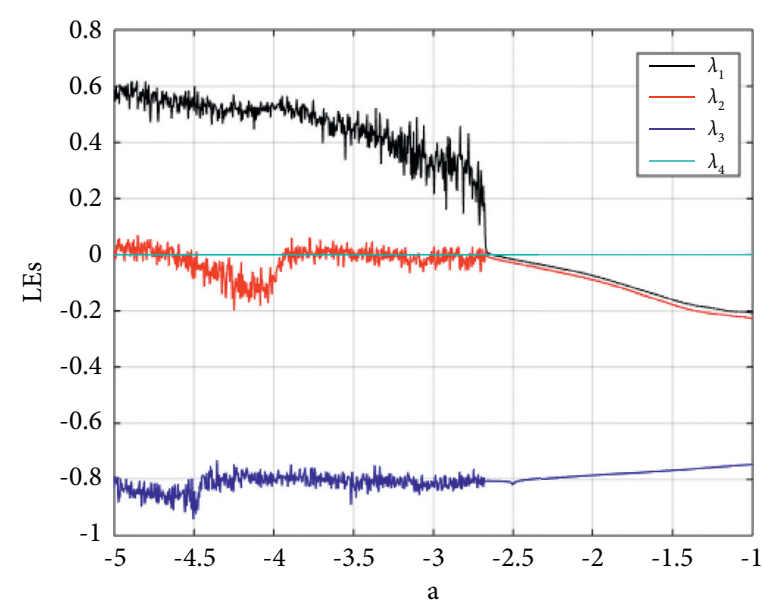

(a)

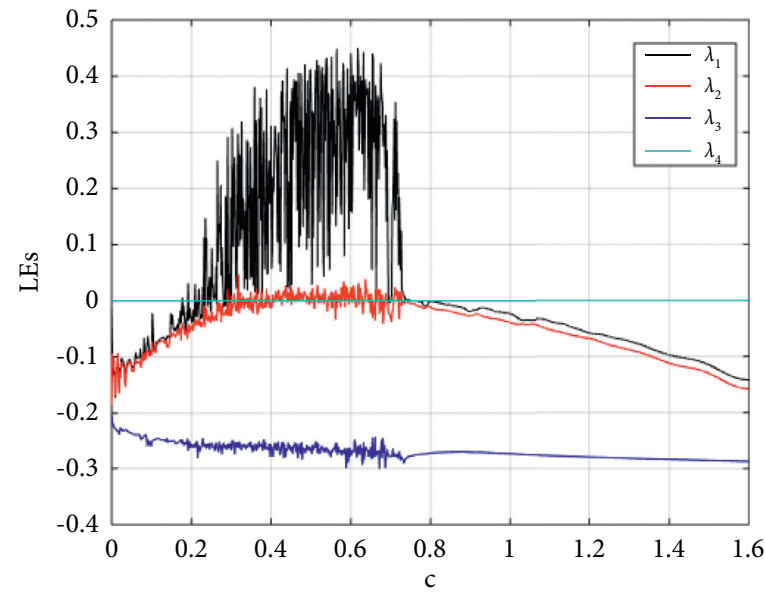

(c)

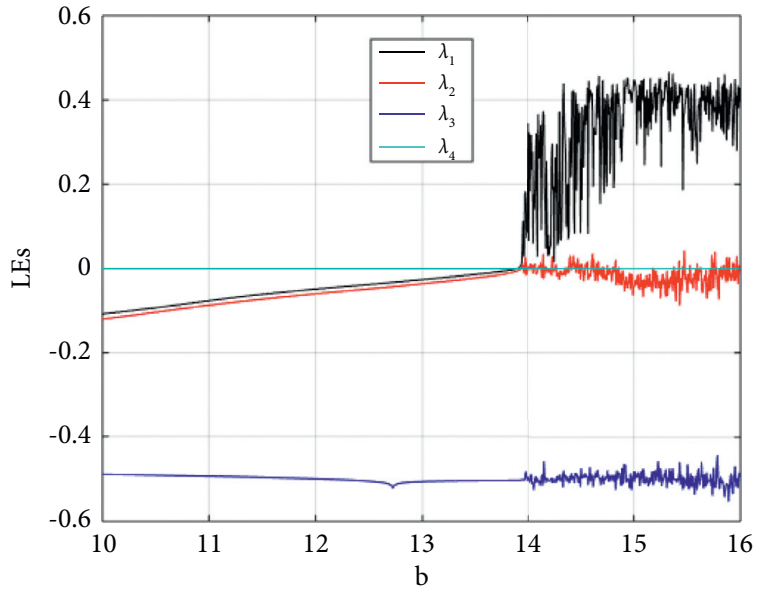

(b)

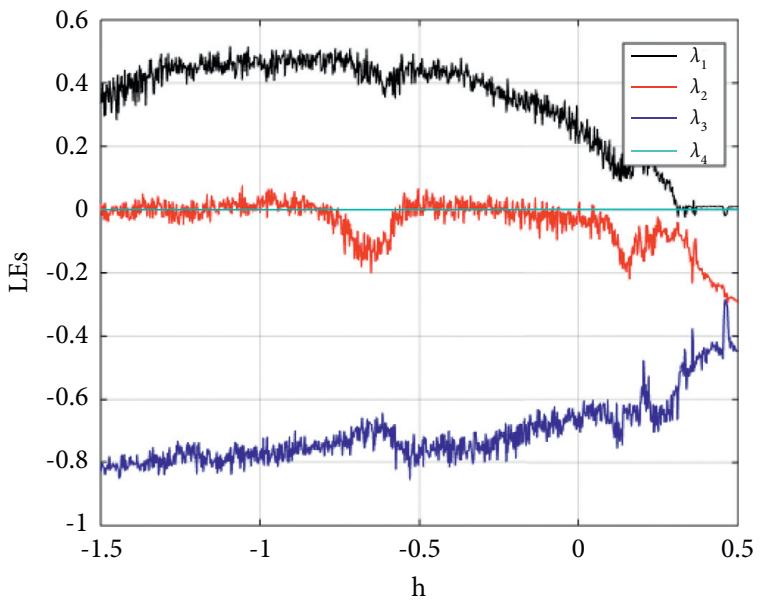

(d)

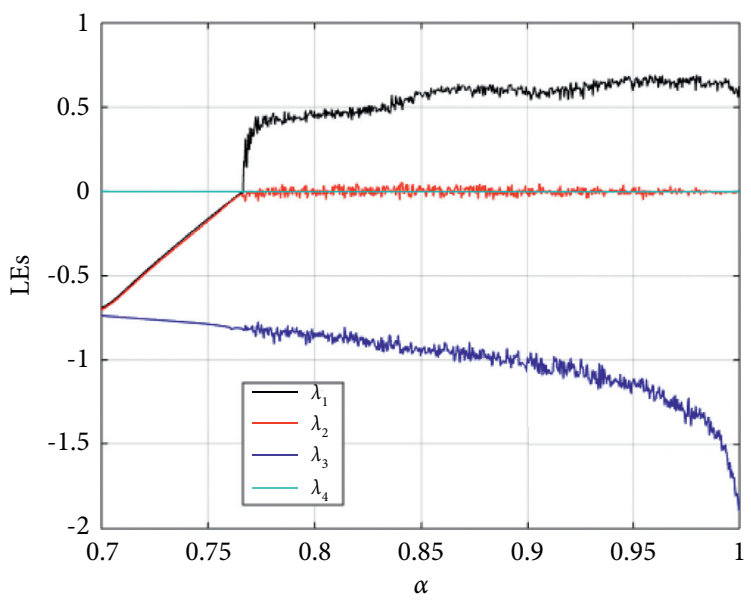

(e)

FIGURE 6: Lyapunov spectrum of system (4) with fixing the parameter values according to the set $A_{1} A_{1}$, (a) except varying the parameter $a$ and setting $\alpha=0.77 \alpha=0.77$, (b) except varying the parameter $b$ and setting $\alpha=0.77$, (c) except varying the parameter $c$ and setting $\alpha=0.77$, (d) except varying the parameter $h$ and setting $\alpha=0.77$, and (e) varying $\alpha$. 


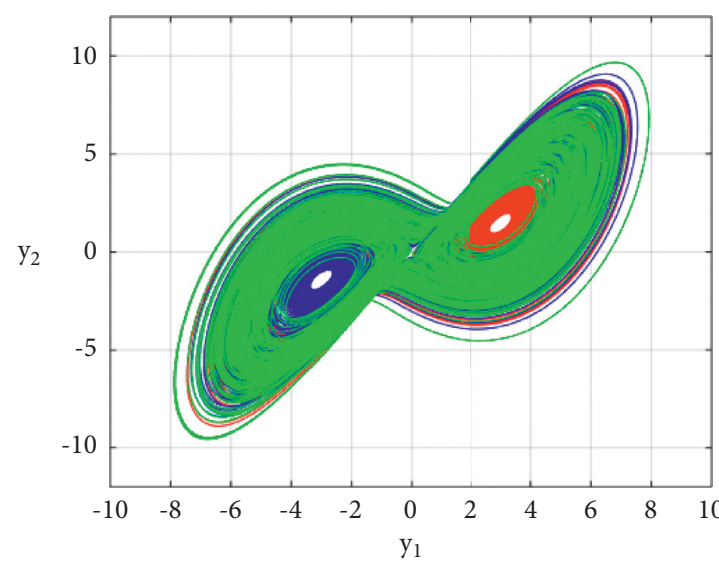

(a)

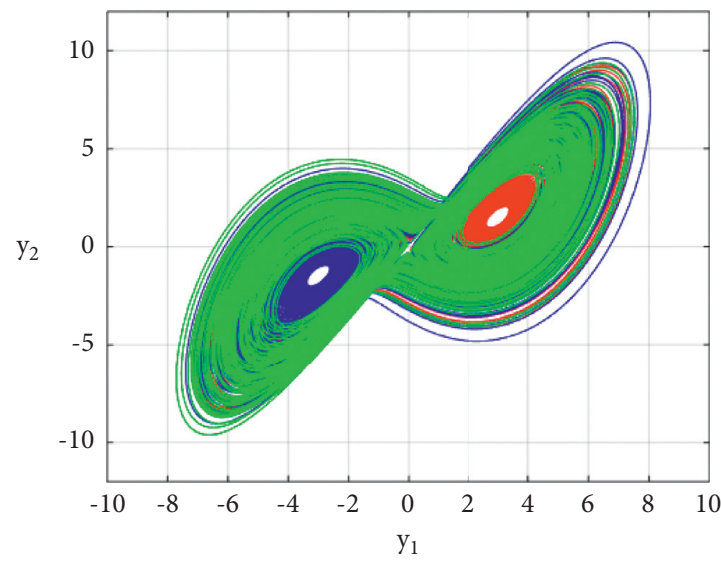

(c)

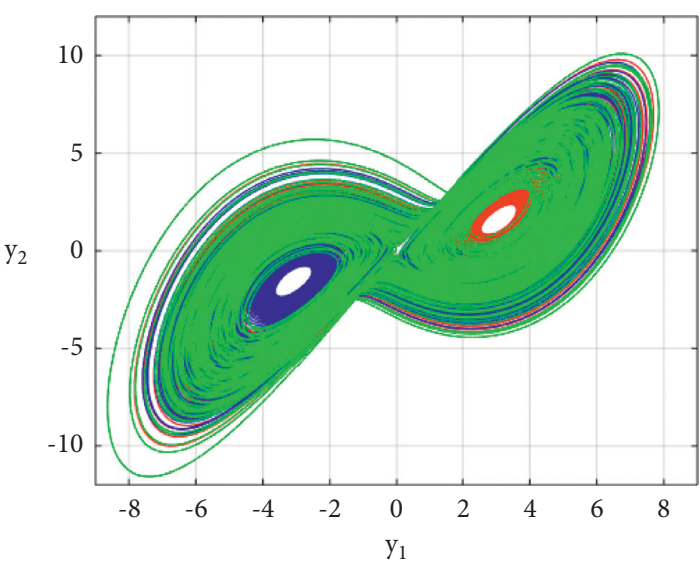

(b)

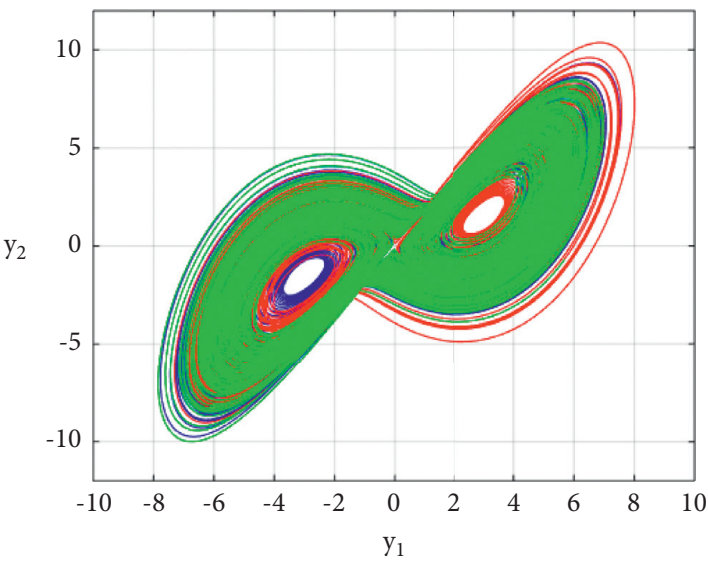

(d)

Figure 7: The projection of the self-excited attractor of the fractional-order Matouk's system in the $y_{1} y_{2}-$ plane with initial conditions $(0.1,0,1,0.01)$ for green trajectory; $(3,1,15,0.0199)$ for red trajectory; and $(-3,-1,15,0.0199)$ for blue trajectory and using the following parameter values: (a) $a=-3.2, b=15, c=0.6, d=-0.0001, h=-1.5, \alpha=0.77$; (b) $a=-3, b=15.75, c=0.6, d=-0.0001, h=-1.5, \alpha=0.77$; (c) $a=-3, b=15, c=0.6, d=-0.0001, h=-1.46, \alpha=0.77$; (d) $a=-3, b=15, c=0.6, d=-0.0001, h=-1.5, \alpha=0.775$.

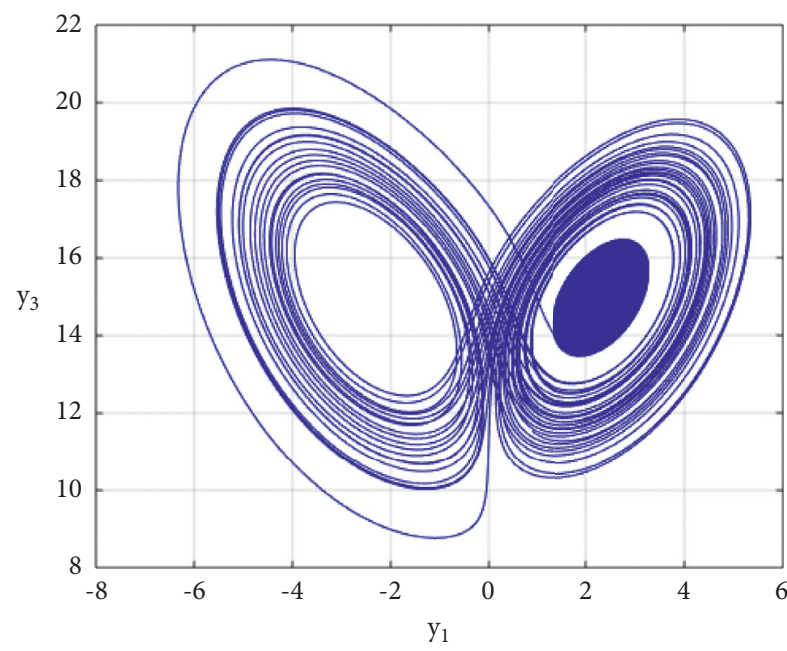

(a)

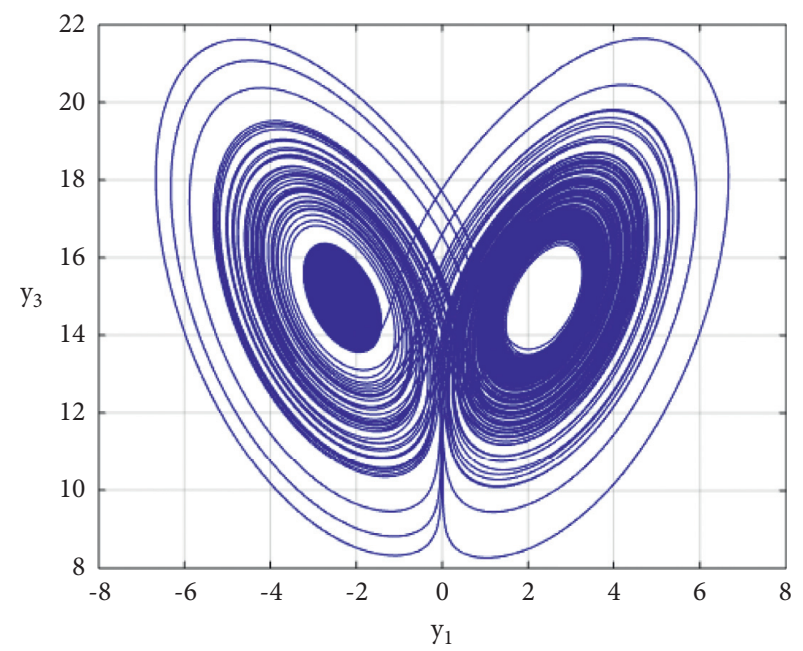

(b)

FIgURE 8: The one-eyed face self-excited chaotic attractor of the fractional-order Matouk's system in the $y_{1} y_{3}-$ plane with initial conditions $(0.01,0.01,0.01,0.01), a=-3, b=15, d=-0.0001, h=-1.5, \alpha=0.77$, and $(\mathrm{a}) c=0.34$ and $(\mathrm{b}) c=0.35$. 


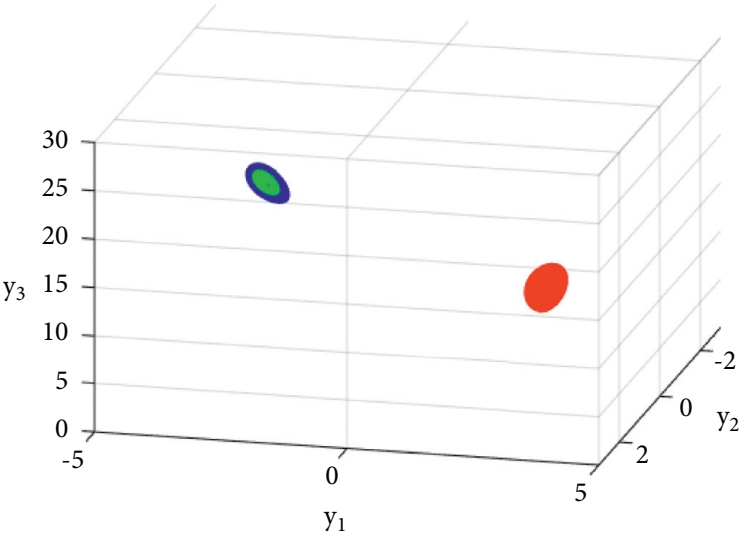

(a)

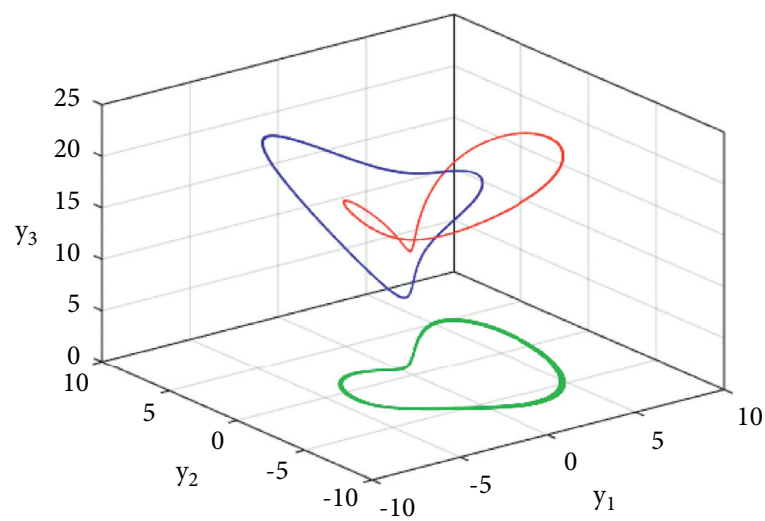

(c)

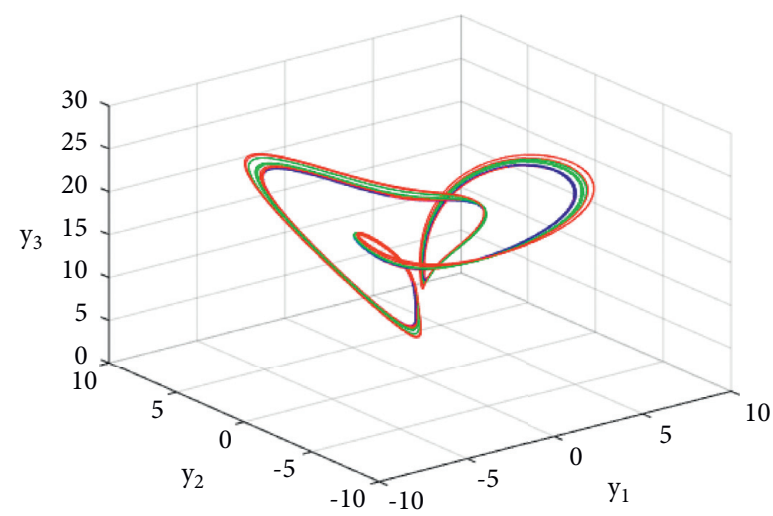

(b)

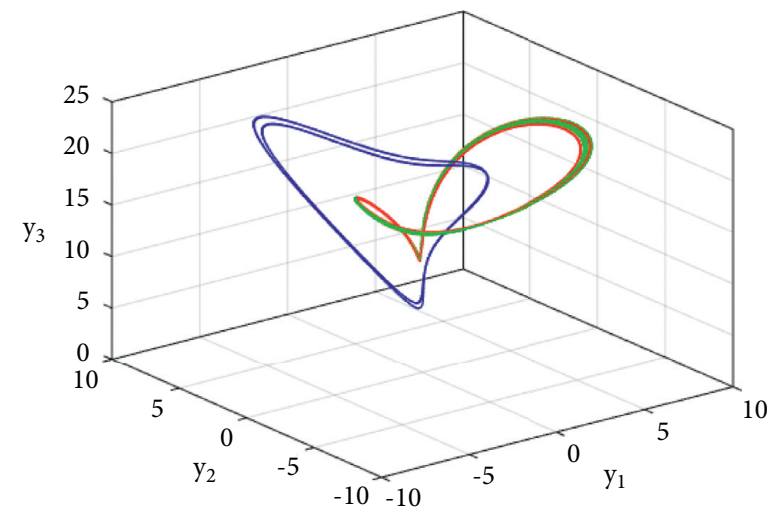

(d)

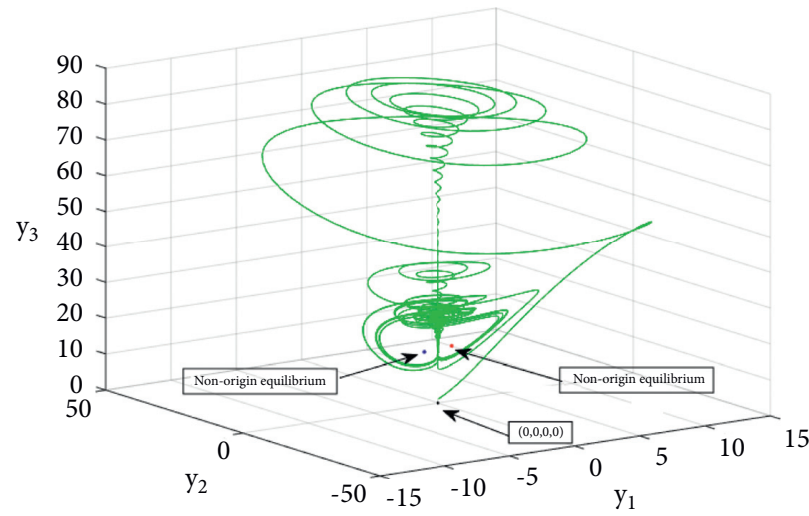

(e)

Figure 9: 3D view of multihidden attractors in the fractional-order Matouk's system, with the fractional parameter $\alpha=0.77$ and initial conditions $(0.1,0,1,0.01)$ for green trajectory, $(3,1,15,0.0199)$ for red trajectory, and $(-3,-1,15,0.0199)$ for blue trajectory, showing the following: (a) coexisting unstable saddle-focus attractors for $a=-3, b=15, c=0.8, d=-0.0001, h=-1.5$; (b) coexisting homoclinic orbits for $a=-3, b=15, c=0.6, d=-0.0001, h=0.0 .18$; (c) coexisting invariant closed curves for $a=-3, b=15, c=0.6, d=-0.0001, h=0.3$; (d) coexisting higher periodic orbits and homoclinic orbits for $a=-3, b=15, c=0.6, d=-0.0001, h=0.25$; and (e) existence of strange hidden chaotic attractor in the integer-order Matouk's system, with $a=-3, b=15, c=0.1, d=-1, h=-1.5$ using initial conditions $(0.1,0,1,0.01)$ for green trajectory. 

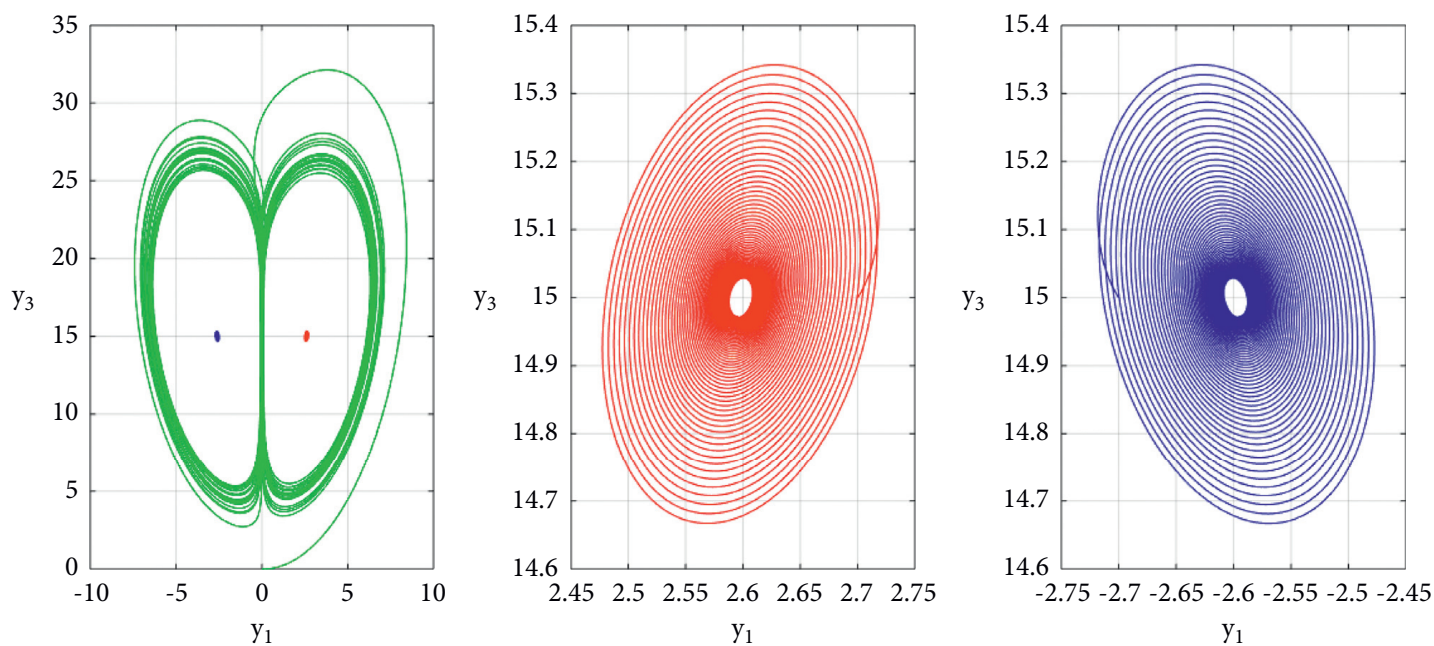

Figure 10: View of the integer-order Matouk's system (4) in the $y_{1} y_{3}$-plane with parameter selection $A_{2}$, showing the hidden chaotic attractor (green plot), one-point attractor corresponding to $E q^{(2)}=(2.5981,10.1,15,0)$ (red plot), and one-point attractor corresponding to $E q^{(3)}=(-2.5981,-10.1,15,0)$ (blue plot).
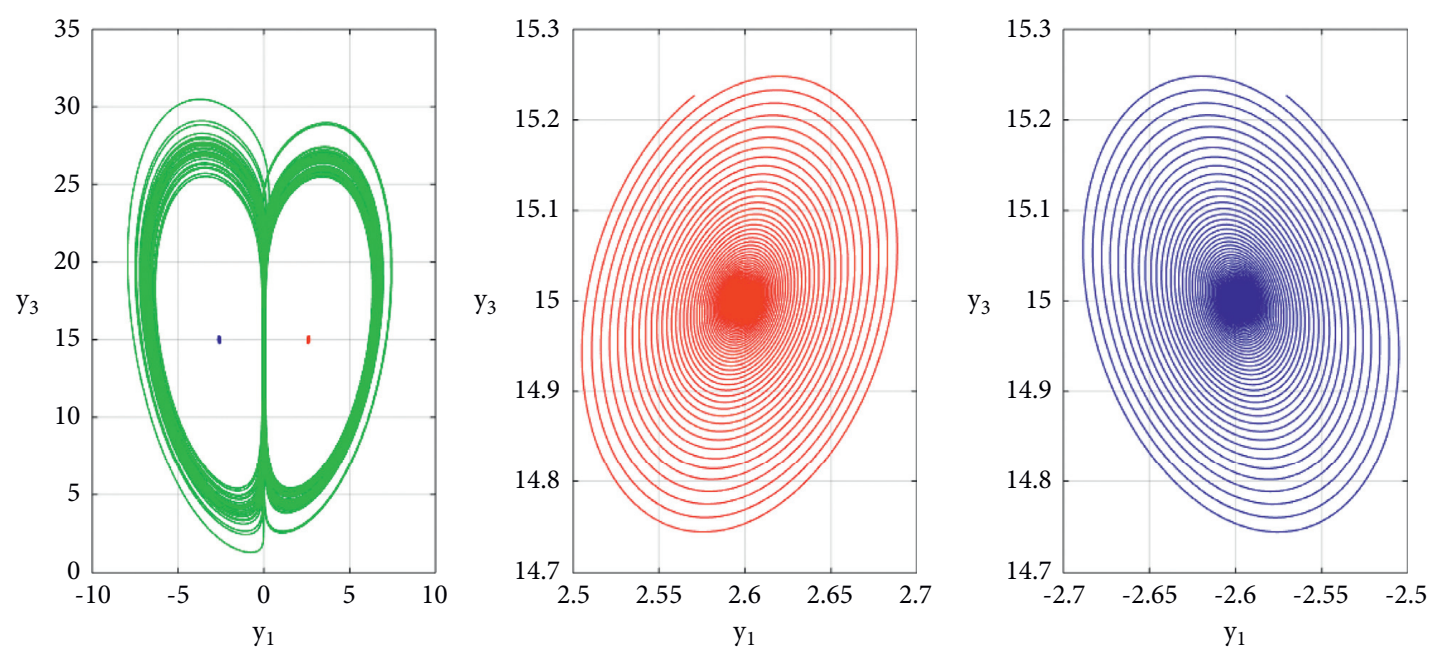

Figure 11: View of Matouk's system (4) in $y_{1} y_{3}$-plane with parameter selection $A_{2}$ and $\alpha=0.998$, showing the hidden chaotic attractor (green plot), one-point attractor corresponding to $E q^{(2)}=(2.5981,10.1,15,0)$ (red plot), and one-point attractor corresponding to $E q^{(3)}=$ $(-2.5981,-10.1,15,0)$ (blue plot).

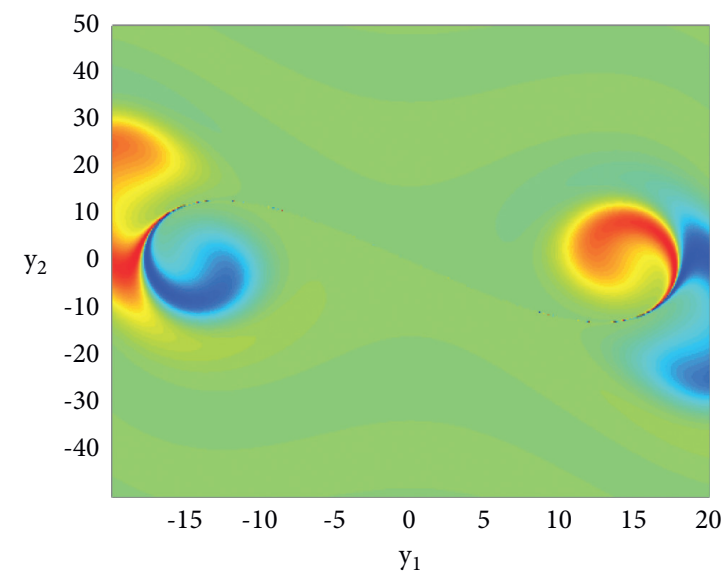

FIgURE 12: A cross section for $y_{3}=0$ and $y_{4}=0$ of three colored basins of attraction for the hidden chaotic attractor (green), the equilibrium $E q^{(2)}$ (orange), and the equilibrium $E q^{(3)}$ (turquoise). 


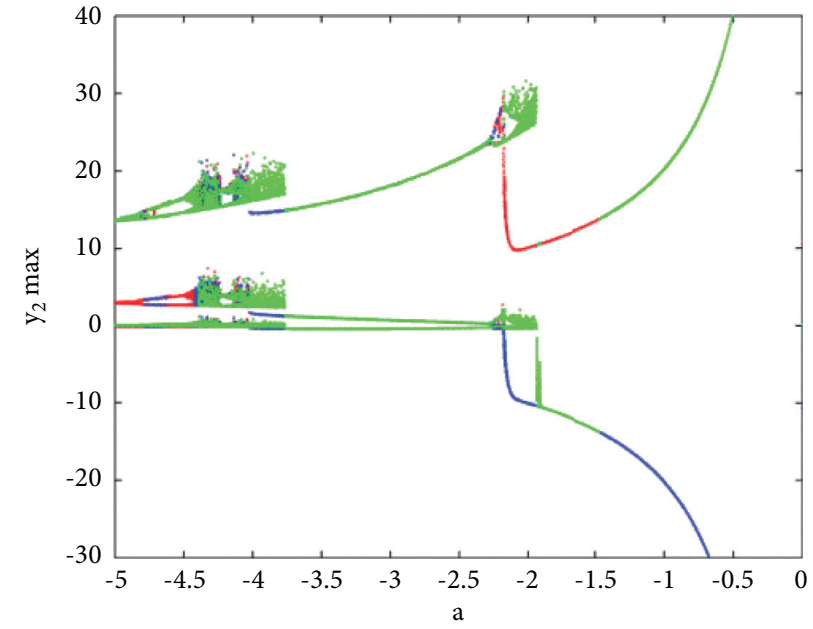

(a)

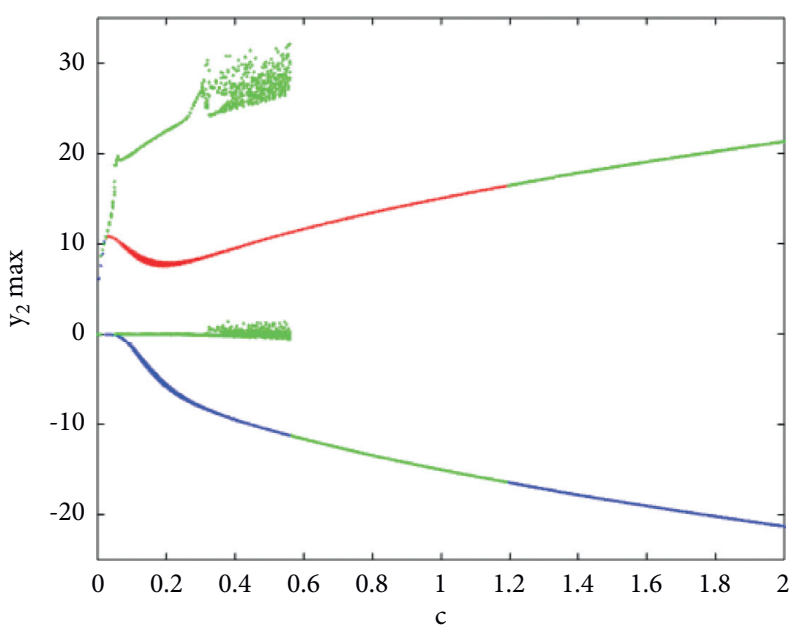

(c)

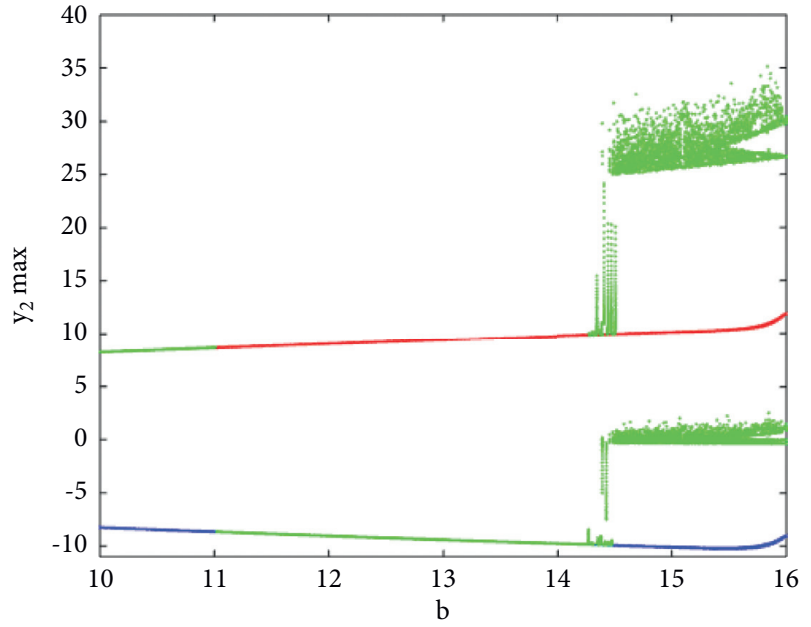

(b)

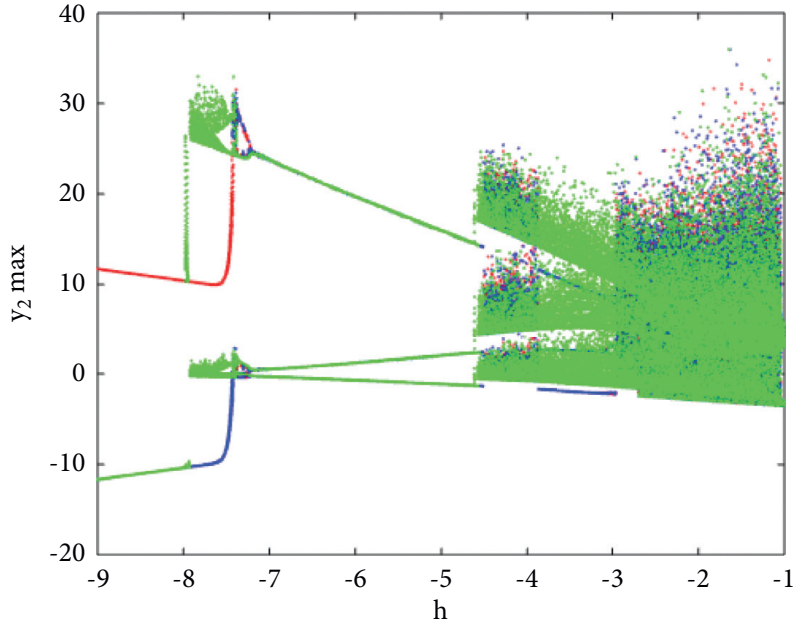

(d)

FIGURE 13: The bifurcation diagrams of the integer-order Matouk's system with varying one parameter and fixing the others according to the set $A_{2}$ and using initial conditions $(0.01,0.01,0.01,0.01)$ (green domain), $(2.7,10.6,15,0.0001)$ (red domain), and $(-2.7,-10.6,15,0.0001)$ (blue domain).

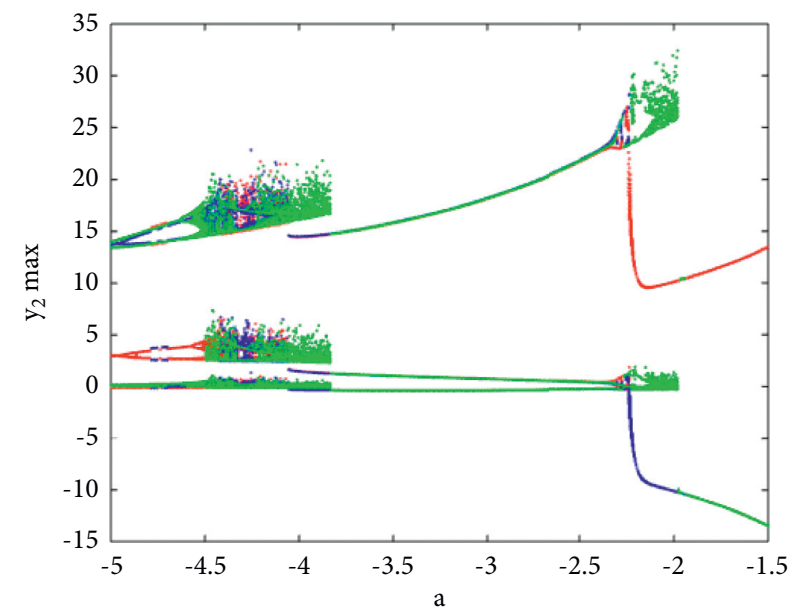

(a)

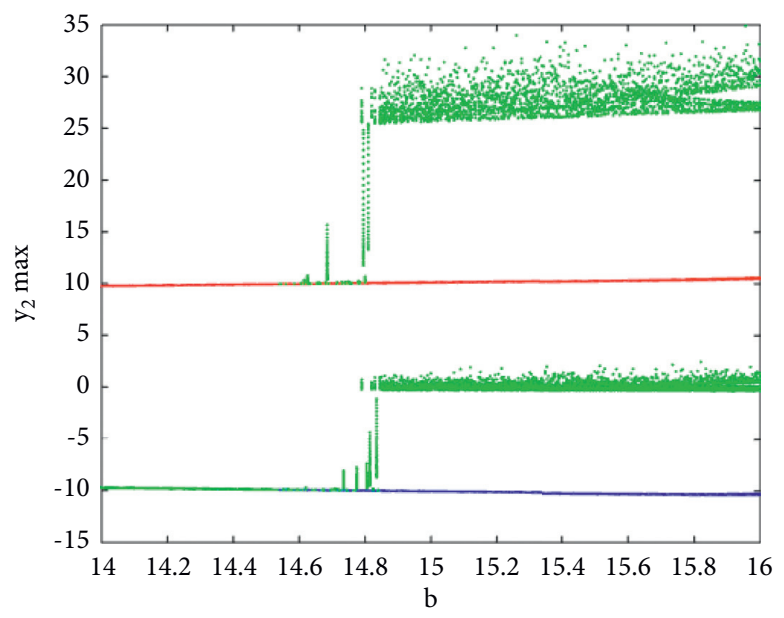

(b)

Figure 14: Continued. 


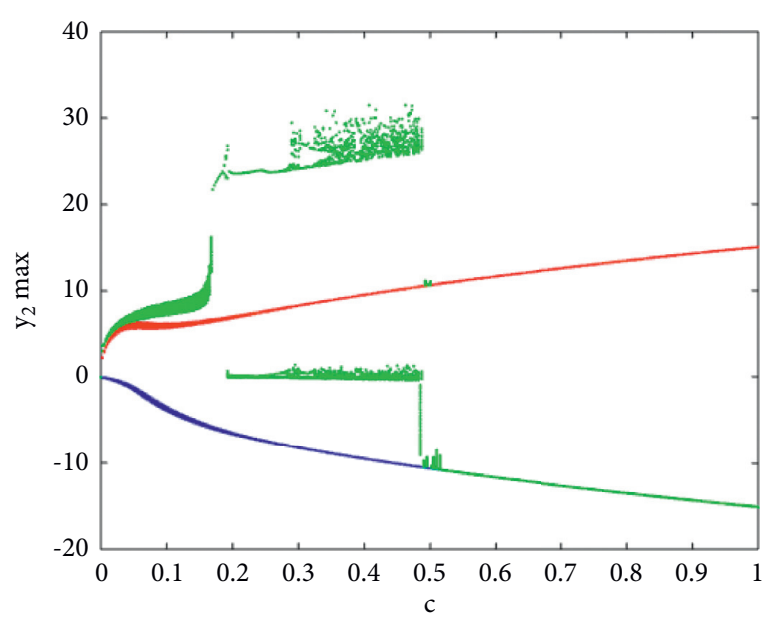

(c)

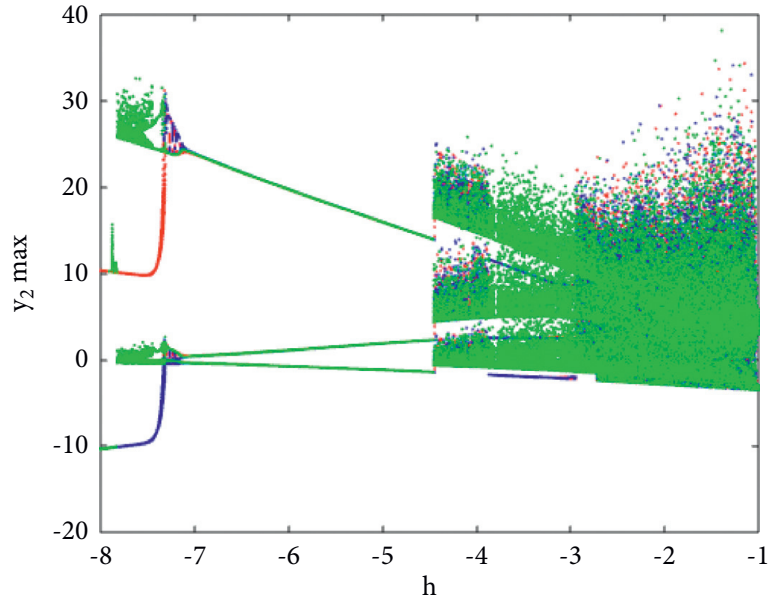

(d)

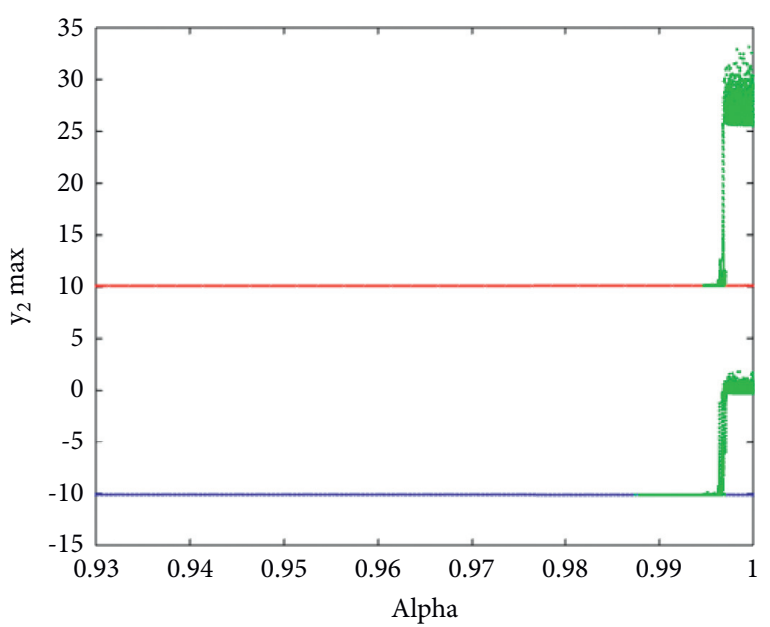

(e)

FIgURE 14: The bifurcation diagrams of the fractional-order Matouk's system with $\alpha=0.998$, varying one parameter and fixing the others according to the set $A_{2}$ and using initial conditions (0.01, 0.01,0.01,0.01) (green domain), $(2.7,10.6,15,0.0001)$ (red domain), and $(-2.7,-10.6,15,0.0001)$ (blue domain).

\section{Conclusion}

We have obtained a rich variety of complex dynamics including the occurrence of hidden chaotic attractors in a new $4 \mathrm{D}$ system, named Matouk's fractional-order hyperchaotic system. We have introduced two case studies, in which hidden chaotic attractors are found in the fractional-order system, with orders 3.08 and 3.992 , respectively. In the second case, we have shown that hidden chaotic attractors are obtained in both the integer and fractional-order Matouk's systems. In addition, self-excited chaotic attractors have been obtained in the fractional-order system and its integer-order form. Moreover, the coexistence of multihidden attractors has been shown in the integer-order Matouk's system and its fractional version. The obtained results verify that the fractional form is a better candidate to discuss the complex dynamics of Matouk's hyperchaotic system since it exhibits a variety of complex dynamics including self-excited and hidden chaotic attractors because of its higher degrees of freedom.

Numerical simulations, based on the PECE algorithm, have been used to confirm these important foundations and successful results through the reported strange attractors, computations of bifurcation diagrams, Lyapunov exponents, and basins of attractions. Thus, the system shows a variety of rich dynamics especially chaotic attractors that still exist in this system for a wide dynamical range as the derivative order is varied $(0.77 \leq \alpha \leq 1)$ in some cases. Also, the existence of new shaped attractors in this simple $4 \mathrm{D}$ system has been depicted such as the existence of the one-eyed face selfexcited chaotic attractors. Consequently, the system has been shown to be a new chaotic system according to the basic criteria in the literature.

Future work may investigate the analytical conditions for the existence of Shil'nikov's chaos in the fractional-order case. 


\section{Data Availability}

The data sets generated during the current study are available from the corresponding author on reasonable request.

\section{Conflicts of Interest}

The authors declare that there are no conflicts of interest.

\section{Acknowledgments}

This work has been supported by Scientific Research Deanship at University of Ha'il, Ha'il, Saudi Arabia, through Project Number RG-21067.

\section{References}

[1] A. M. Sayed and A. E. Matouk, "Sanjay Kumar, Vakkar Ali, and Lahcene Bachioua, "Chaotic dynamics and chaos control in a fractional-order satellite model and its time-delay counterpart," Discrete Dynamics in Nature and Society, vol. 2021, Article ID 5542908, 11 pages, 2021.

[2] J. Wang, L. Xiao, K. Rajagopal, A. Akgul, S. Cicek, and B. Aricioglu, "Fractional-order analysis of modified Chua's circuit system with the smooth degree of 3 and its microcontroller-based implementation with analog circuit design," Symmetry, vol. 340, p. 13, 2021.

[3] Z. Li, W. Ma, and N. Ma, "Partial topology identification of tempered fractional-order complex networks via synchronization method," Mathematical Methods in the Applied Sciences, pp. 1-14, 2021.

[4] A. E. Matouk, "Complex dynamics in susceptible-infected models for COVID-19 with multi-drug resistance," Chaos, Solitons \& Fractals, vol. 140, Article ID 110257, 2020.

[5] A. E. Matouk and I. Khan, "Complex dynamics and control of a novel physical model using nonlocal fractional differential operator with singular kernel," Journal of Advanced Research, vol. 24, pp. 463-474, 2020.

[6] E. Ahmed and A. E. Matouk, "Complex dynamics of some models of antimicrobial resistance on complex networks," Mathematical Methods in the Applied Sciences, vol. 44, no. 2, pp. 1896-1912, 2021.

[7] S. Kumar, A. E. Matouk, H. Chaudhary, and S. Kant, "Control and synchronization of fractional-order chaotic satellite systems using feedback and adaptive control techniques," International Journal of Adaptive Control and Signal Processing, vol. 35, no. 4, pp. 484-497, 2021.

[8] W. W. Mohammed, E. S. Aly, and A. E. Matouk, "An analytical study of the dynamic behavior of Lotka-Volterra based models of COVID-19," Results in Physics, vol. 26, Article ID 104432, 2021.

[9] A. Othman Almatroud, "Synchronisation of two different uncertain fractional-order chaotic systems with unknown parameters using a modified adaptive sliding-mode controller," Advances in Difference Equations, vol. 2020, p. 78, 2020.

[10] A. Zenkour and A. Ahmed, "Fractional thermoelasticity model of a 2D problem of mode-I crack in a fibre-reinforced thermal environment," J. Appl. Comput. Mech, vol. 5, no. 2, pp. 269-280, 2019.

[11] A. Ahmed and H. Ahmad, "A modified thermoelastic fractional heat conduction model with a single-lag and two different fractional orders," J. Appl. Comput. Mech, vol. 7, no. 3, pp. 1676-1686, 2021.

[12] G. A. Leonov, N. V. Kuznetsov, and V. I. Vagaitsev, "Hidden attractor in smooth Chua systems," Physica D: Nonlinear Phenomena, vol. 241, no. 18, pp. 1482-1486, 2012.

[13] M.-F. Danca, "Hidden chaotic attractors in fractional-order systems," Nonlinear Dynamics, vol. 89, no. 1, pp. 577-586, 2017.

[14] N. N. Bautin, "On the number of limit cycles generated on varying the coefficients from a focus or centre type equilibrium state," Doklady Akademii Nauk SSSR, vol. 24, no. 7, pp. 668-671, 1939.

[15] N. V. Kuznetsov, O. A. Kuznetsova, and G. A. Leonov, "Visualization of four normal size limit cycles in two-dimensional polynomial quadratic system," Differential equations and dynamical systems, vol. 21, no. 1-2, pp. 29-34, 2013.

[16] D. Hilbert, "Mathematical problems," Bulletin of the American Mathematical Society, vol. 8, pp. 437-479, 1901.

[17] Q. Yang, Z. Wei, and G. Chen, "An unusual 3D autonomous quadratic chaotic system with two stable node-foci," International Journal of Bifurcation and Chaos, vol. 20, no. 4, pp. 1061-1083, 2010.

[18] D. Dudkowski, S. Jafari, T. Kapitaniak, N. V. Kuznetsov, G. A. Leonov, and A. Prasad, "Hidden attractors in dynamical systems," Physics Reports, vol. 637, pp. 1-50, 2016.

[19] B. Munmuangsaen and B. Srisuchinwong, "A hidden chaotic attractor in the classical Lorenz system," Chaos, Solitons \& Fractals, vol. 107, pp. 61-66, 2018.

[20] N. V. Kuznetsov and G. A. Leonov, "Hidden attractors in dynamical systems: systems with no equilibria, multistability and coexisting attractors," IFAC Proceedings Volumes, vol. 47, no. 3, pp. 5445-5454, 2014.

[21] N. V. Kuznetsov, "Hidden attractors in fundamental problems and engineering models: a short survey," AETA 2015: Recent Advances in Electrical Engineering and Related Sciences, vol. 371, pp. 13-25, 2016.

[22] J. P. Singh and B. K. Roy, "The simplest 4-D chaotic system with line of equilibria, chaotic 2-torus and 3-torus behaviour," Nonlinear Dynamics, vol. 89, no. 3, pp. 1845-1862, 2017.

[23] J. P. Singh and B. K. Roy, "A more chaotic and easily hardware implementable new 3-D chaotic system in comparison with 50 reported systems," Nonlinear Dynamics, vol. 93, no. 3, pp. 1121-1148, 2018.

[24] A. E. Matouk, "Dynamics and control in a novel hyperchaotic system," International Journal of Dynamics and Control, vol. 7, no. 1, pp. 241-255, 2019.

[25] A. E. Matouk, "A novel fractional-order system: chaos, hyperchaos and applications to linear control," J. Appl. Comput. Mech, vol. 7, no. 2, pp. 701-714, 2021.

[26] M. Caputo, "Linear models of dissipation whose Q is almost frequency independent--II," Geophysical Journal International, vol. 13, no. 5, pp. 529-539, 1967.

[27] R. A. Van Gorder, "Shil'nikov chaos in the 4D Lorenz-Stenflo system modeling the time evolution of nonlinear acousticgravity waves in a rotating atmosphere," Nonlinear Dynamics, vol. 72, no. 4, pp. 837-851, 2013.

[28] C. P. Silva, "Shil'nikov's theorem-a tutorial," IEEE Transactions on Circuits and Systems I: Fundamental Theory and Applications, vol. 40, no. 10, pp. 675-682, 1993.

[29] P. Prakash, K. Rajagopal, J. P. Singh, and B. K. Roy, "Megastability in a quasi-periodically forced system exhibiting multistability quasi-periodic behaviour, and its analogue circuit simulation," International Journal of Electronics and Communications, vol. 92, 2018. 
[30] K. Diethelm, N. J. Ford, and A. D. Freed, "A predictor-corrector approach for the numerical solution of fractional differential equations," Nonlinear Dynamics, vol. 29, no. 3-22, 2002.

[31] K. Diethelm, "An algorithm for the numerical solution of differential equations of fractional order," Electronic Transactions on Numerical Analysis, vol. 5, no. 1-6, 1997.

[32] F. Nazarimehr, B. Saedi, S. Jafari, and J. Sprott, "Are perpetual points sufficient for locating hidden attractors?" Int. J. Bifurc. Chaos, vol. 27, no. 3, Article ID 1750037, 2017.

[33] D. Dudkowski, A. Prasad, and T. Kapitaniak, "Perpetual points: new tool for localization of coexisting attractors in dynamical systems," Int. J. Bifurc. Chaos, vol. 27, no. 4, Article ID 1750063, 2017.

[34] J. C. Sprott, "A proposed standard for the publication of new chaotic systems," Int. J. Bifurc. Chaos, vol. 21, no. 9, pp. 2391-2394, 2011. 